\title{
REVISÃO DA LITERATURA SOBRE O TRATAMENTO FARMACOLÓGICO DOS TRANSTORNOS ALIMENTARES
}

\section{LITERATURE REVIEW ON PHARMACOLOGICAL TREATMENT OF EATING DISORDERS}

Jacqueline Gabriele Soares Ferreira1, Chrislen Nascimento de Oliveira², Jhéssyca Glaycianne Souza das Chagas $^{3}$, Gleicy Kelly China Quemel ${ }^{4}$, Natasha Costa da Rocha Galucio ${ }^{5}$, Danilo Reymão Moreira ${ }^{6}$

\section{RESUMO}

Os distúrbios alimentares são doenças de caráter psicopatológico e quando não tratadas corretamente prejudicam o indivíduo afetando-o socialmente, mentalmente e biologicamente. Dentro desse contexto, o trabalho avaliou, com base na literatura, os fármacos utilizados no tratamento dos transtornos alimentares. A metodologia utilizada foi a revisão integrativa da literatura com apoio da análise documental de Bardin. Para a busca das literaturas foram utilizados descritores, contidos nos DECs: [Transtornos da compulsão alimentar/Binge-Eating Disorder], [Tratamento Farmacológico/Drug therapy], [Bulimia/bulimia] e [Anorexia/anorexia]. Para coleta de dados, foram utilizadas literaturas encontradas na base de dado MEDLINE, na biblioteca do SCIELO e em repositórios. Foram selecionadas 22 literaturas, sendo que 63,6\% (14) são da biblioteca do SCIELO, 18,2\% (4) da base de dados MEDLINE e 18,2\% (4) de repositórios de Ensino Superior. Foi observado que o uso de medicamento é essencial quando a terapia não medicamentosa não surtir efeito, uso de fármacos a Fluoxetina, Imipramina e Sibutramina são as principais medicações para o tratamento. Além disso, o tratamento dos transtornos alimentares exige uma equipe multidisciplinar para auxiliar no processo, com isto, o profissional farmacêutico exerce uma função primordial que garante um diagnóstico favorável e um tratamento adequado. Portanto, medicamento como o dimesilato de lisdexanfetamina, utilizado no tratamento de lúpus, entre outros fármacos para tratar outras doenças podem ser de suma importância para o tratamento dos transtornos alimentares, quando associados a outras terapias não farmacológica e do acompanhamento com profissionais especializados.

PALAVRAS-CHAVE: Transtornos Alimentares. Farmacoterapia. Psicofarmacologia

\section{ABSTRACT}

Eating disorders are psychopathological diseases and when not treated correctly they affect the individual, affecting him socially, mentally and biologically. Within this context, the study evaluated, based on the literature, the drugs used in the treatment of eating disorders. The methodology used was an integrative literature review supported by Bardin's document analysis. To search the literature, descriptors were used, contained in the DECs: [Binge Eating Disorders/Binge-Eating

\footnotetext{
1 Graduada em Bacharelado em Farmácia pela Escola Superior da Amazônia (ESAMAZ) - Belém-PA e Farmacêutica Responsável Técnica (RT) da Rede de Farmácia Droga Norte - Belém-Pa. ORCID: https://orcid.org/0000-0002-7040-7899

2 Graduada em Bacharelado em Farmácia pela Escola Superior da Amazônia (ESAMAZ) - Belém-PA. ORCID: https://orcid.org/0000-0001-9342-431X

${ }^{3}$ Graduada em Bacharelado em Farmácia pela Escola Superior da Amazônia (ESAMAZ) - Belém-PA. ORCID: https://orcid.org/0000-0001-8295-9888

${ }^{4}$ Licenciada Plena em Ciências com habilitação em química (UFPA), mestre em Ciências Ambientais (UEPA) e docente do curso de Farmácia da Escola Superior da Amazônia (ESAMAZ) - Belém-PA. ORCID: https://orcid.org/0000-0003-1280-560X

5 Doutoranda em Genética e Biologia Molecular (UFPA), mestre em Ciências Farmacêuticas (UFPA), especialista em Vigilância em Saúde (Sírio-Libanês) e em Gestão da Assistência Farmacêutica (UFSC). Coordenadora do Curso de Graduação em Bacharelado em Farmácia da Escola Superior da Amazônia (ESAMAZ). ORCID: https://orcid.org/0000-0003-4923-1478

6 Doutor em Biologia dos Agentes infecciosos e Parasitários (UFPA), mestre em doenças tropicais (UFPA), especialista em farmacologia Clínica (Centro Universitário Internacional), Farmacêutico da Polícia Militar e Docente do curso de Farmácia da Escola Superior da Amazônia (ESAMAZ) - Belém-PA. ORCID: https://orcid.org/0000-0003-0381-5064
}

RECIMA21 - Ciências Exatas e da Terra, Sociais, da Saúde, Humanas e Engenharia/Tecnologia 


\section{RECIMA21 - REVISTA CIENTÍFICA MULTIDISCIPLINAR ISSN 2675-6218}

REVISÃO DA LITERATURA SOBRE O TRATAMENTO FARMACOLÓGICO DOS TRANSTORNOS ALIMENTARES Jacqueline Gabriele Soares Ferreira, Chrislen Nascimento de Oliveira, Jhéssyca Glaycianne Souza das Chagas, Gleicy Kelly China Quemel, Natasha Costa da Rocha Galucio, Danilo Reymão Moreira

Disorder], [Pharmacological Treatment/Drug Therapy], [Bulimia/bulimia] and [Anorexia/anorexia]. For data collection, literature found in the MEDLINE database, in the SCIELO library and in repositories were used. 22 literatures were selected, of which 63.6\% (14) are from the SCIELO library, $18.2 \%$ (4) from the MEDLINE database and 18.2\% (4) from Higher Education repositories. It was observed that the use of medication is essential when non-drug therapy does not work, the use of drugs such as Fluoxetine, Imipramine and Sibutramine are the main medications for the treatment. In addition, the treatment of eating disorders requires a multidisciplinary team to assist in the process, with this, the pharmacist plays a key role that ensures a favorable diagnosis and adequate treatment. Therefore, medication such as lisdexamphetamine dimesylate, used in the treatment of lupus, among other drugs to treat other diseases, can be of paramount importance for the treatment of eating disorders, when associated with other non-pharmacological therapies and monitoring with specialized professionals.

\section{KEYWORDS: Eating Disorders. Pharmacotherapy. Psychopharmacology}

\section{INTRODUÇÃO}

O transtorno da compulsão alimentar está associado ao ato de comer compulsivamente uma quantidade de comida muito maior do que se está acostumado, havendo ou não a necessidade fisiológica de comer (DE AZEVEDO; DOS SANTOS; DA FONSECA, 2004).

As diferenças entre exagero alimentar, compulsão e obesidade são noções básicas que transpassam o trajeto das psicopatologias alimentares e causam dúvidas e confusão até mesmo entre profissionais de saúde. Os distúrbios alimentares são caracterizados como transtornos alimentares (TA) de cunho psicológico, que afetam o bem-estar físico, social e neurobiológico, e são classificados como: transtorno emocional de evitação de comida, síndrome de repulsa generalizada, comer seletivo, anorexia nervosa (NA), bulimia nervosa (BN) transtorno da compulsão alimentar (TCA) e transtorno da compulsão alimentar periódica (TCAP) (VIANNA, 2018).

Os quadros de depressão e ansiedade abrem as portas para os transtornos alimentares assim como para outras patologias. Em alguns indivíduos estes transtornos desencadeiam síndromes menstruais, osteoporose, arritmia cardíaca, além desses distúrbios o consumo de alimentos inadequados faz com que haja uma alteração nos micronutrientes podendo assim ocasionar um quadro anêmico. Alguns estudos envolvendo pacientes obesos demonstram uma forte relação positiva e complementar entre compulsão alimentar, vício em comida e transtornos depressivos. A presença de qualquer uma dessas comorbidades é considerada um fator de risco para instalação de novas doenças (DOS ANJOS et al., 2020).

Várias terapias podem ser utilizadas para o tratamento dos transtornos como o uso de tratamento que não envolva substâncias químicas, dentre estas terapias podemos encontrar o uso da aromaterapia, acupuntura, práticas de exercícios físicos como ioga entre outras. Entretanto, nem sempre o uso de terapias paliativas é o suficiente para a regressão de episódios compulsivos, sendo necessária uma abordagem medicamentosa, analisando sempre o comportamento do indivíduo quanto as suas ações, para que este não tenha ações consideradas de risco ligadas aos distúrbios, podendo ser prejudiciais a sua saúde (COMIN; SANTOS, 2012). 


\section{RECIMA21 - REVISTA CIENTÍFICA MULTIDISCIPLINAR ISSN 2675-6218}

REVISÃO DA LITERATURA SOBRE O TRATAMENTO FARMACOLÓGICO DOS TRANSTORNOS ALIMENTARES Jacqueline Gabriele Soares Ferreira, Chrislen Nascimento de Oliveira, Jhéssyca Glaycianne Souza das Chagas, Gleicy Kelly China Quemel, Natasha Costa da Rocha Galucio, Danilo Reymão Moreira

Além de desencadear patologias, o quadro de TA está muito interligado ao momento atual em que nos encontramos, assim podemos relacionar a Covid-19 com hábitos alimentares inadequados, pois com a pandemia muitos sofreram uma brusca mudança em suas rotinas, desta forma o isolamento social pode ocasionar em muitas pessoas quadros psicopatológicos, conduzindo a um consumo excessivo de alimentos (WERNECK et al., 2020).

Diante do exposto, o presente trabalho avaliou, por meio da literatura, os principais fármacos utilizados no tratamento dos transtornos alimentares, assim como o papel do farmacêutico dentro do tratamento dos transtornos alimentares, identificando quais os possíveis fatores associados aos TA e a outras doenças.

\section{METODOLOGIA}

A metodologia escolhida para a pesquisa foi uma Revisão Integrativa da Literatura (RIL), cuja finalidade é agregar e reduzir resultados de pesquisas de uma determinada temática ou questão, de maneira ordenada e sistemática de modo a construir bases de conhecimento sólido com relação ao tema (MENDES; SILVEIRA; GALVÃO, 2008).

A pergunta que norteou o trabalho foi "Quais os fármacos mais utilizados no tratamento dos transtornos alimentares?". A busca pela literatura se deu em Repositórios de Ensino Superior, na Scientific Electronic Library Online (SCIELO) e na base de dados Medical Literature Analysis and Retrieval System Online (MEDLINE).

Os descritores utilizados, contidos nos Descritores em Ciências da Saúde (DECs), foram: [Transtornos da compulsão alimentar/Binge-Eating Disorder], [Tratamento Farmacológico/Drug therapy], [Bulimia/bulimia] e [Anorexia/anorexia] combinados ou não com o operador booleano "and"

Quanto os critérios utilizados, os de inclusão foram literaturas completas, disponíveis no idioma português, inglês e espanhol, publicadas no período de 2011 a abril de 2021; e exclusas literaturas duplicadas e com acesso restrito ao pagamento.

A análise crítica dos estudos foi realizada segundo a análise de conteúdo de Bardin (2011), dividida em três fases: na primeira, a pré-análise, foram utilizados cinco filtros em conformidade com os critérios de inclusão e exclusão para a seleção dos estudos para a próxima fase. A segunda fase, denominada exploração do material, foi realizada a categorização em eixos temáticos, sendo esses: 1- medicamentos utilizados no tratamento da compulsão alimentar; 2- atuação do farmacêutico; 3 fatores associados ao comportamento de risco da compulsão alimentar; e 4 - a correlação com outras patologias. $\mathrm{Na}$ terceira fase, a interpretação dos resultados obtidos, consistiu na apresentação dos estudos selecionados e explanação das categorias de discussão.

\section{RESULTADOS E DISCUSSÃO}

A pesquisa selecionou 22 literaturas, sendo 13,6\% (3) dos artigos no idioma em inglês e $86,4 \%$ (19) em português. Com relação a busca das literaturas 63,6\% (14) são da biblioteca do SCIELO, 18, 2 (4) da base de dados MEDLINE e 18,2\% (4) de Repositório de Ensino Superior. 


\section{RECIMA21 - REVISTA CIENTÍFICA MULTIDISCIPLINAR ISSN 2675-6218}

REVISÃO DA LITERATURA SOBRE O TRATAMENTO FARMACOLÓGICO DOS TRANSTORNOS ALIMENTARES Jacqueline Gabriele Soares Ferreira, Chrislen Nascimento de Oliveira, Jhéssyca Glaycianne Souza das Chagas, Gleicy Kelly China Quemel, Natasha Costa da Rocha Galucio, Danilo Reymão Moreira

Quanto à abordagem dos estudos: $13,64 \%$ (3) discorrem sobre COVID-19 e compulsão alimentar, 4,53\% (1) relatam sobre a psicanálise e compulsão alimentar, 27,27\% (6) referente ao tratamento farmacológico da compulsão alimentar, 13,64\% (3) a importância da atenção farmacêutica direcionada a compulsão alimentar, 13,64\% (3) tratam do diagnóstico, avaliação e intervenção no transtorno da compulsão alimentar, 13,64\% (3) abordam os fatores associados ao comportamento de risco da compulsão alimentar e os outros $13,64 \%$ (3) relatam sobre as possíveis patologias associadas aos transtornos de compulsão alimentar.

Os estudos selecionados estão dispostos no quadro 1, organizados cronologicamente, que são apresentados Título/Autor/ ano, objetivo, metodologia e conclusão dos estudos

Quadro 1: Estudos Selecionados

\begin{tabular}{|c|c|c|c|c|}
\hline № & $\begin{array}{c}\text { TÍTULO/ } \\
\text { AUTOR/ANO }\end{array}$ & OBJETIVO & METODOLOGIA & CONCLUSÃO \\
\hline 01 & $\begin{array}{l}\text { Uma revisão dos } \\
\text { estudos latino- } \\
\text { americanos sobre } \\
0 \quad \text { transtorno da } \\
\text { compulsão } \\
\text { alimentar } \\
\text { periódica. } \\
\text { PALAVRAS et al. } \\
\text { (2011) }\end{array}$ & $\begin{array}{l}\text { Revisar o estado } \\
\text { de arte da literatura } \\
\text { latino-americana } \\
\text { sobre o transtorno } \\
\text { da compulsão } \\
\text { alimentar } \\
\text { periódica. }\end{array}$ & $\begin{array}{l}\text { Busca literária sobre } \\
\text { estudos realizados em } \\
\text { países latino-americanos } \\
\text { usando-se o termo "binge } \\
\text { eating", que deveriam } \\
\text { preencher critérios } \\
\text { parciais ou completos do } \\
\text { transtorno da compulsão } \\
\text { alimentar periódica pelo } \\
\text { Manual Diagnóstico e } \\
\text { Estatístico de Transtornos } \\
\text { Mentais-4a Edição }\end{array}$ & $\begin{array}{l}\text { O transtorno da compulsão } \\
\text { alimentar periódica mostra-se } \\
\text { um fenômeno verificável na } \\
\text { América Latina com } \\
\text { características clínicas } \\
\text { semelhantes às encontradas } \\
\text { na literatura internacional. } \\
\text { Esta revisão fornece subsídios } \\
\text { para que o transtorno da } \\
\text { compulsão alimentar periódica } \\
\text { seja considerado uma } \\
\text { categoria distinta de } \\
\text { transtorno alimentar na } \\
\text { Classificação Internacional de } \\
\text { Doencas-11ä Edição. }\end{array}$ \\
\hline 02 & $\begin{array}{lr}\text { Classificação } & \text { e } \\
\text { Diagnóstico } & \text { de } \\
\text { Transtornos } & \\
\text { Alimentares } & \text { na } \\
\text { Infância: } & \text { Nem } \\
\text { DSM, nem CID-10 } \\
\\
\text { PINHEIRO (2011) }\end{array}$ & $\begin{array}{l}\text { Revisar a Tabela } 2 \\
-\quad \text { Descritores e } \\
\text { operador booleano } \\
\text { do estado atual } \\
\text { dos critérios } \\
\text { diagnósticos dos } \\
\text { TAS infantis de } \\
\text { acordo } \quad \text { com } \\
\text { sistemas } \\
\text { classificação de } \\
\text { tradicionais }\end{array}$ & $\begin{array}{l}\text { Foram revistos todos os } \\
\text { critérios de cada tipo de } \\
\text { transtorno e } \\
\text { paralelamente à sua } \\
\text { indicação são apontados } \\
\text { os principais pontos } \\
\text { frágeis e de críticas. }\end{array}$ & $\begin{array}{l}\text { Futuras investigações devem } \\
\text { focalizar o aperfeiçoamento } \\
\text { dos critérios diagnósticos } \\
\text { infantis para que seja possível } \\
\text { auxiliar especialistas na } \\
\text { detecção e tratamento de } \\
\text { cada transtorno. }\end{array}$ \\
\hline 03 & $\begin{array}{l}\text { Uma análise } \\
\text { psicanalítica da } \\
\text { compulsão e da } \\
\text { impulsão a partir } \\
\text { da perspectiva do } \\
\text { gozo e do ato. } \\
\text { BITTENCOURT; } \\
\text { FONTENELE } \\
\text { (2013) }\end{array}$ & 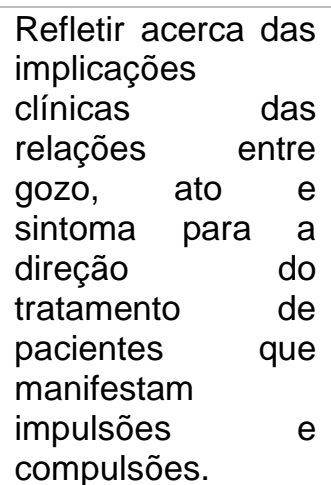 & $\begin{array}{l}\text { Foi realizado um } \\
\text { levantamento das } \\
\text { publicações brasileiras, } \\
\text { hospedadas nas bases de } \\
\text { dados INDEXPSI, } \\
\text { PePSIC, SCIELO, LILACS } \\
\text { e Portal Nacional BVS } \\
\text { Brasil em Saúde, }\end{array}$ & $\begin{array}{l}\text { Esse trabalho visa suscitar } \\
\text { uma discussão concernente à } \\
\text { compulsão e à impulsão com } \\
\text { relação ao diagnóstico } \\
\text { psiquiátrico de compulsão } \\
\text { alimentar, pois acredita-se que } \\
\text { um analista, ao demarcar a } \\
\text { diferença entre tais conceitos, } \\
\text { pode trazer contribuições } \\
\text { relevantes ao diagnóstico. }\end{array}$ \\
\hline
\end{tabular}




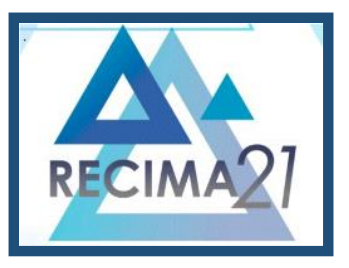

\section{RECIMA21 - REVISTA CIENTÍFICA MULTIDISCIPLINAR ISSN 2675-6218}

REVISÃO DA LITERATURA SOBRE O TRATAMENTO FARMACOLÓGICO DOS TRANSTORNOS ALIMENTARES ( (a)

$04 \begin{aligned} & \text { Fatores } \\ & \text { associados ao } \\ & \text { comportamento } \\ & \text { alimentar } \\ & \text { inadequado em } \\ & \text { adolescentes } \\ & \text { escolares. }\end{aligned}$

FORTES MORGADO; FERREIRA (2013)

05 O que é risco

transtornos

alimentares

adolescentes?

LEAL et al. (2013)

06 Fatores associados ao comportamento de risco para transtornos alimentares adolescentes cidade de São Paulo.

LEAL (2013)

\section{Acolhimento}

vínculo em serviço assistência portadores transtornos alimentares.

RAMOS; PEDRÃO (2013) em

de

a de

\section{Associar IC, grau} de comprometimento psicológico ao exercício (GCPE), adiposidade

corporal

(AC), estado nutricional (EN), NE e etnia ao CAI em adolescentes

Caracterizar comportamento de risco transtornos alimentares (TA) e sua frequência entre adolescentes em uma revisão da literatura nacional e internacional.

\section{Identificar}

prevalência comportamentos de risco para em transtornos

na alimentares,

ăo práticas saudáveis controle do peso e fatores associados em adolescentes.

e Avaliar

um acolhimento área e usuários de servido de assistência multidisciplinar em pacientes com Transtornos Alimentares.

\begin{tabular}{|c|c|}
\hline $\begin{array}{ll}\begin{array}{l}\text { Efetividade } \\
\text { intervenções }\end{array} & \text { de } \\
\text { psicossociais } & \text { em } \\
\text { transtornos } & \\
\text { alimentares: } & \text { um } \\
\text { panorama } & \text { das } \\
\text { revisões } & \\
\text { sistemáticas } & \\
\text { Cochrane. } & \\
& \\
\text { COSTA; MELNIK } \\
\text { (2016) }\end{array}$ & $\begin{array}{l}\text { Compilar } \\
\text { achados } \\
\text { trabalhos } \\
\text { científicos } \\
\text { relevantes, com } \\
\text { estudos } \\
\text { controlados } \\
\text { randomizados, } \\
\text { para promover } \\
\text { conhecimento } \\
\text { sobre a efetividac } \\
\text { de intervençõe } \\
\text { psicossociais e } \\
\text { transtornos }\end{array}$ \\
\hline
\end{tabular}

Este estudo apresentou delineamento transversal e foi realizado no ano de 2011, teve seu início após sua aprovação pelo Comitê de Ética e Pesquisa em Seres Humanos da UFJF.

Foi realizada uma busca bibliográfica por meio de uma revisão integrativa nas bases de dados PubMed e Lilacs e no portal SciELO

Trata-se de um estudo transversal, onde foi realizada uma busca bibliográfica por meio de uma revisão integrativa nas bases de dados Pubmed, Lilacs, Scielo.

Utilizou se uma

e abordagem metodológica de cunho qualitativo de quarta geração.

Foi realizada uma revisão sistemática da literatura.
Pode-se concluir que 0 comportamento alimentar inadequado esteve associado à insatisfação corporal e ao grau de comprometimento psicológico ao exercício no sexo feminino.

Diferentes nomenclaturas e instrumentos são utilizados para avaliar comportamento de risco para TA entre adolescentes, com grande amplitude nos resultados de prevalência.

Foi encontrada uma ampla variação na maneira de caracterizar o comportamento de risco para o TA, assim como nos tipos de instrumentos e nomenclaturas utilizados. Os comportamentos de risco para TA foram caracterizados principalmente pela presença dos sintomas clássicos.

Conclui-se que na perspectiva de usuários e profissionais que buscam atendimento, priorizam um atendimento humanizado.

Os estudos mostraram que a abordagem cognitivocomportamental é a modalidade de intervenção psicológica mais efetiva. Outras intervenções que demonstraram efetividade foram a terapia comportamental dialética, terapia interpessoal, intervenções de base familiar e terapias de apoio. A autoajuda baseada em manual é uma intervenção 


\section{RECIMA21 - REVISTA CIENTÍFICA MULTIDISCIPLINAR ISSN 2675-6218}

REVISÃO DA LITERATURA SOBRE O TRATAMENTO FARMACOLÓGICO DOS TRANSTORNOS ALIMENTARES ( Gleicy Kelly China Quemel, Natasha Costa da Rocha Galucio, Danilo Reymão Moreira
(a)

alimentares.

09 A Novel Nociceptin Receptor

Antagonist

LY2940094 Inhibits

Excessive Feeding

Behavior

Rodents:

Possible

Mechanism for the

Treatment of Binge

Eating Disorder

\section{STATNICK et al.} (2016)

10 A contribuição do farmacêutico para a promoção do acesso e uso racional de medicamentos essenciais no SUS.

DE MELO; DE CASTRO (2017) nociceptina-

A transtornos alimentares
Abordar o uso de opioide-peptídeo

(NOP) como possível escolha de tratamento para

$\begin{array}{lr}\text { Descrever } & \text { o } \\ \text { processo } & \text { da } \\ \text { inserção } & \text { do } \\ \text { farmacêutico } & \text { na } \\ \text { equipe de uma } \\ \text { Unidade Básica de } \\ \text { Saúde e r os } \\ \text { resultados } & \text { na } \\ \text { promoção } & \text { do } \\ \text { acesso e uso } \\ \text { racional } \\ \text { medicamentos. }\end{array}$

11 A eficácia do mindful eating para transtornos alimentares obesidade: revisão integrativa.

\section{ALMEIDA;} ASSUNPÇÃO (2018)

12 Avaliação intervenção transtorno compulsão alimentar uma (TCA): sistemática. revisão

CAUDURO; PAZ; PACHECO (2018) O objetivo deste artigo é identificar a eficácia da e intervenção mindful eating para 0 tratamento do transtorno de compulsão alimentar (TCA). \begin{tabular}{r|lr} 
e & Este artigo \\
no & objetivou identificar
\end{tabular} da as características metodológicas dos estudos, descrever as técnicas de intervenção e os instrumentos de avaliação utilizados no tratamento do
Utilizou se um estudo

Trata-se de estudo
descritivo, transversal,
realizado em unidade de
atenção primária do
município de São Paulo.
Trata-se de estudo
descritivo, transversal,
realizado em unidade de
atenção primária do
município de São Paulo. TCA integrativa da literatura. e Scielo, PubMed, Psyclnfo e Science Direct para consulta. qualitativo e in vitro.

frequentemente efetiva, e
pode ser oferecida de
diferentes formas para
prevenção e tratamento dos
transtornos alimentares.

Conclui-se que LY2940094 pode ser útil no tratamento de distúrbios do comportamento apetitivo, tais

como transtorno da compulsão alimentar periódica, escolha de alimentos e alimentação excessiva, que

levar à obesidade e suas complicações médicas associadas e morbidade.

Apesar das barreiras iniciais, com a integração do farmacêutico à equipe multiprofissional, ele passa a assumir papel relevante na redução de problemas relacionados a medicamentos e na melhoria da qualidade das prescrições médicas. A presença do farmacêutico na unidade para a realização das intervenções foi de fundamental importância para - alcance de resultados positivos.

Foi realizada uma revisão Conclui-se que por meio dessa revisão, verificou-se a necessidade da realização de estudos que avaliem a eficácia e a efetividade do tratamento de TCA, bem como estudos de seguimento que avaliem a manutenção ou não dos benefícios advindos do tratamento.

Foi conduzida uma Essa revisão sistemática, teve revisão sistemática da como objetivo descrever literatura de estudos instrumentos de avaliação de empíricos nacionais e TCA e as técnicas internacionais, utilizando empregadas nas intervenções as bases de dados Lilacs psicológicas realizadas com portadores de Transtorno da

Compulsão Alimentar. Os resultados sugerem um interesse crescente e global por esse tema, tanto no que diz respeito ao diagnóstico 


\section{RECIMA21 - REVISTA CIENTÍFICA MULTIDISCIPLINAR ISSN 2675-6218}

REVISÃO DA LITERATURA SOBRE O TRATAMENTO FARMACOLÓGICO DOS TRANSTORNOS ALIMENTARES Jacqueline Gabriele Soares Ferreira, Chrislen Nascimento de Oliveira, Jhéssyca Glaycianne Souza das Chagas, Gleicy Kelly China Quemel, Natasha Costa da Rocha Galucio, Danilo Reymão Moreira

13 Tratamento transtornos alimentares: perfil dos pacientes desfecho seguimento

MANOCHIO et al. (2018) Traçar o perfil e
desfecho Avaliação por meio de uma amostragem tratamento em quantitativa e qualitativa, pacientes, a fim de possibilitar reflexões sobre e indicar um bom do diagnóstico. serviço existente.
14 Atenção farmacêutica no tratamento transtornos alimentares

\section{RODRIGUES} (2018)

15 Consumo formulações emagrecedoras e risco de transtornos alimentares em universitários cursos de saúde.

Avaliar o consumo de formulações emagrecedoras e sua possível associação com dia 0 de
Retratar a prática da atenção farmacêutica no tratamento transtornos alimentares

de

(da

Trata-se de um estudo de revisão bibliográfica, a partir de literaturas específicas. As bases de dados utilizadas foram o Scielo, Lilacs, dados disponibilizados pelo Ministério da Saúde e Organização Mundial de Saúde e Diretrizes oficiais. Utilizou se um estudo transversal para investigar - consumo de formulações emagrecedoras.

SILVA et al. (2018)

\section{Transtorno}

Compulsão

Alimentar: Revisão

Sistemática da

Literatura

BLOC et al. (2019)

\section{Objetivam escrever e discutir a produção científica sobre a compulsão alimentar entre os anos de 2006 e 2016.} Alimentares (TAs)

\section{Revisão sistemática da literatura (RSL) de caráter qualitativo.}

17 Based Cognitive Behavioral Therapy via Videoconference For Patients With Bulimia Nervosa

O objetivo da pesquisa foi avaliar a viabilidade da ICBT via videoconferência para pacientes quanto às intervenções.

Concluiu-se que os pacientes atendidos pelo serviço são em sua maioria, mulheres jovens apresentando anorexia nervosa e também foi encontrada elevada taxa de abandono ao tratamento. Estudos como este são importantes para auxiliar profissionais na busca de recursos para melhores resultados nos acompanhamentos desses quadros clínicos, a fim de minimizar o alto índice de abandono e promover melhores resultados de recuperação clínica.

Ressalta a importância da orientação farmacêutica visando a melhora do paciente com um prognóstico favorável.

Conclui-se que o consumo de formulações está associado tanto a presença de riscos para TA quanto para os níveis sociais e econômicos.

\section{Foram analisados neste estudo os elementos que contribuíram para a construção de um transtorno que implica em um grande sofrimento para os sujeitos. Conforme observado nesta revisão, o adoecimento perpassa diferentes vieses e não se restringe unicamente a uma esfera corporal ou psicológica.}

ICBT via videoconferência é viável em pacientes japoneses com bulimia nervosa e transtorno da compulsão alimentar periódica. 


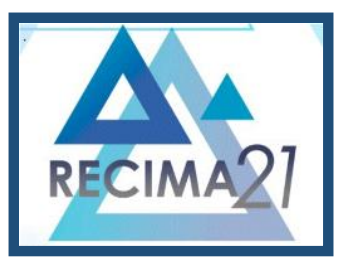

\section{RECIMA21 - REVISTA CIENTÍFICA MULTIDISCIPLINAR ISSN 2675-6218}

REVISÃO DA LITERATURA SOBRE O TRATAMENTO FARMACOLÓGICO DOS TRANSTORNOS ALIMENTARES A Gleicy Kelly China Quemel, Natasha Costa da Rocha Galucio, Danilo Reymão Moreira

and Binge-Eating com

Disorder: Pilot

Prospective Single-

Arm Feasibility

Trial.

HAMATANI et al. (2019)

18

Avaliação do
consumo alimentar
em
pacientes rom
diagnóstico de
depressão e/ou
ansiedade

ARAÙJO et al. (2020)

19 COVID-19 e os impactos na saúde mental: uma amostra do Rio Grande do Sul, Brasil

DUARTE et al. (2020)

21 Polaprezinc (ZincL-Carnosine

Complex) as an Add-on Therapy for Binge Disorder and Bulimia Nervosa, and the Possible Involvement of Zinc

Comportamental.

nervosa ou

transtorno da

compulsão

alimentar

periódica.

Busca relacionar
O estudo método transversal retrospectivo. utilizou 0 exploratório e alguns nutrientes imprescindíveis na biossíntese de moléculas associadas ao quadro de depressão e ansiedade.

Verificar os fatores associados indicadores sintomas transtornos mentais residentes do Rio Grande do Sul, durante o período inicial da política de distanciamento social decorrente da pandemia da COVID-19.

\section{Discorrer sobre} impacto

pandemia

da

na

saúde mental dos universitários e na educação médica. de

de

residentes no Estado do Rio Grande do Sul.
Trata-se de uma revisão de literatura realizada nas seguintes bases de dados: SciELO, LILACS e MEDLINE.
Como os estudantes de Medicina apresentam incertezas sobre o futuro de sua formação em decorrência dessas transformações, são submetidos a uma carga emocional que causa/deflagra danos à saúde mental deles. Existem ainda dúvidas sobre os reflexos desse contexto no período "pós-Covid" e seus impactos na educação médica, assim como sobre a manutenção de medidas adotadas em tempos de crise.

Esses achados oferecem evidências preliminares para o efeito eficácia do polaprezinco no tratamento de TCAP e BN e sugerir o envolvimento de deficiência de zinco nessas condições. 


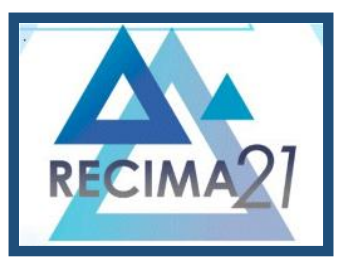

\section{RECIMA21 - REVISTA CIENTÍFICA MULTIDISCIPLINAR ISSN 2675-6218}

REVISÃO DA LITERATURA SOBRE O TRATAMENTO FARMACOLÓGICO DOS TRANSTORNOS ALIMENTARES Jacqueline Gabriele Soares Ferreira, Chrislen Nascimento de Oliveira, Jhéssyca Glaycianne Souza das Chagas, Gleicy Kelly China Quemel, Natasha Costa da Rocha Galucio, Danilo Reymão Moreira

\begin{tabular}{|c|c|c|c|c|}
\hline & $\begin{array}{l}\text { Deficiency in } \\
\text { These Conditions. } \\
\text { A Pilot Study } \\
\text { SAKAE; SUKA; } \\
\text { YANAGISAWA } \\
(2020)\end{array}$ & & & \\
\hline 22 & \begin{tabular}{lr} 
Mudanças & de \\
comportamentos & \\
saudáveis durante \\
a quarentena por \\
conta da pandemia \\
do COVID-19 entre \\
6.881 & adultos \\
brasileiros & com \\
depressão & e \\
35.143 & sem \\
depressão. & \\
\multicolumn{3}{c}{ WERNECK et al. } \\
(2020)
\end{tabular} & $\begin{array}{lr}\begin{array}{lr}\text { Analisar } \\
\text { associação }\end{array} & \text { entre } \\
\text { depressão } & \\
\text { previamente } & \\
\text { diagnosticada } & \text { e } \\
\text { alterações } & \text { na } \\
\text { atividade } & \text { física } \\
\text { (AF), } & \text { tempo } \\
\text { assistindo } & \text { TV, } \\
\text { consumo de } & \text { frutas } \\
\text { e vegetais, bem } \\
\text { como } & \text { na } \\
\text { frequência } & \text { do } \\
\text { consumo } & \text { de } \\
\text { alimentos } & \\
\text { ultraprocessados } \\
\text { (AUP). }\end{array}$ & $\begin{array}{l}\text { A coleta de dados foi } \\
\text { realizada entre } 24 \text { de abril } \\
\text { e } 24 \text { de maio de } 2020 \text {. } \\
\text { Foram utilizados dados de } \\
41.923 \text { adultos brasileiros } \\
(6.881 \text { com depressão e } \\
35.042 \text { sem depressão) } \\
\text { de uma pesquisa de } \\
\text { comportamentos } \\
\text { âmbito nacional. }\end{array}$ & $\begin{array}{l}\text { Participantes com diagnóstico } \\
\text { prévio de depressão } \\
\text { apresentam maior risco de } \\
\text { incidência de comportamentos } \\
\text { alimentares não saudáveis. }\end{array}$ \\
\hline
\end{tabular}

Fonte: Autores, 2021.

Segundo Ramos e Pedrão (2013) o vínculo com o Serviço de Assistência a Portadores de Transtornos Alimentares, é fundamental, haver uma formação adequada, para desenvolver uma escuta profissional qualificada, responsável por oferecer aos pacientes respostas e resoluções sobre suas necessidades e demandas mais urgentes, a fim de obter um diagnóstico adequado, porém somente a identificação do problema não é suficiente, faz-se necessário uma orientação e indicação de terapia de primeira escolha correta. Está é formada por medicações que buscam reduzir totalmente ou a maior parte do quadro sintomático do paciente.

No contexto dos TA, os profissionais farmacêuticos são de extrema importância tanto na prevenção quanto na identificação de algumas patologias. $O$ contato entre este profissional e o paciente é maior, havendo uma interação que possibilita identificar possíveis diagnósticos como o TA. Quando uma doença é diagnosticada, o farmacêutico pode monitorar o tratamento mais de perto e orientar o uso correto dos medicamentos, observando a eficácia, segurança, reações adversas e ainda tomar medidas não farmacológicas colaborando com a equipe multidisciplinar para possibilitar que o tratamento seja mais eficaz e o prognóstico positivo (RODRIGUES et al, 2020).

O papel associado a assistência farmacêutica no tratamento aos distúrbios alimentares, abrange desde a seleção dos medicamentos até a dispensação correta do mesmo e tem como finalidade garantir o acesso dos pacientes ao medicamento. Por outro lado, cabe a atenção farmacêutica possibilitar um estreitamento do elo farmacêutico-paciente tendo como um dos princípios a qualidade de vida do indivíduo (RODRIGUES, 2018). 


\section{RECIMA21 - REVISTA CIENTÍFICA MULTIDISCIPLINAR ISSN 2675-6218}

Segundo Silva et al. (2018), a etiologia dos transtornos alimentares (TA) é dada a partir de quadros de vulnerabilidade biológica, podendo o indivíduo apresentar predisposições psicólogas e comportamentos de risco como: crises perfeccionistas, exacerbação de exercícios físicos, práticas de dietas restritivas extremas e a indução de episódios de vômito ou uso concomitante de medicamentos laxativos e anorexígenos.

Segundo Guimarães et al. (2019), os mecanismos de diagnósticos dos distúrbios alimentares, podem ser divididos em três categorias: questionários autoaplicáveis, entrevistas clínicas e automonitoramento. Alguns métodos utilizados para a detecção e confirmação dos TA são de extrema importância. O Quadro 2 descreve quais os métodos mais utilizados, o que avaliam, assim como os benefícios e as vantagens.

Quadro 2: Classificação de diagnóstico: dos questionários autoaplicáveis mais utilizados

\begin{tabular}{|c|c|c|}
\hline Nome do método & O que avalia & Benefícios \\
\hline $\begin{array}{c}\text { Eating Attitudes Test ( Teste } \\
\text { de Atitudes Alimentare) }\end{array}$ & $\begin{array}{l}\text { Teste com } 40 \text { questões, } \\
\text { onde cada questão } \\
\text { apresenta seis opções de } \\
\text { resposta. }\end{array}$ & $\begin{array}{l}\text { Indica a presença de padrões } \\
\text { alimentares anormais, porém não } \\
\text { revela a possível psicopatologia }\end{array}$ \\
\hline $\begin{array}{l}\text { Binge Eating Scale (Escala de } \\
\text { compulsão alimentar } \\
\text { periódica) }\end{array}$ & $\begin{array}{l}\text { É composta por } 16 \text { itens, } \\
\text { avalia as manifestações } \\
\text { comportamentais e os } \\
\text { sentimentos e cognições } \\
\text { envolvidas durante } \\
\text { episódios de compulsão } \\
\text { alimentar periódica. }\end{array}$ & $\begin{array}{c}\text { Possibilita a elaboração de } \\
\text { estratégias terapêuticas mais } \\
\text { adequadas e a avaliação sequencial } \\
\text { do tratamento de pacientes obesos. }\end{array}$ \\
\hline $\begin{array}{l}\text { Bulimic Investigatory Test } \\
\text { Edinburgh (Teste de } \\
\text { Investigação Bulímica de } \\
\text { Edimburgo) }\end{array}$ & $\begin{array}{l}\text { é composto por } 30 \\
\text { questões para se } \\
\text { determinar a sintomatologia } \\
\text { relacionada com bulimia, } \\
\text { tendo como respostas "sim" } \\
\text { ou "não". }\end{array}$ & $\begin{array}{l}\text { mede-se a gravidade do } \\
\text { comportamento em função de sua } \\
\text { frequência. }\end{array}$ \\
\hline Body Shape Questionnaire & $\begin{array}{c}\text { Avalia o grau de } \\
\text { insatisfação corporal nas } \\
\text { últimas quatro semanas } \\
\text { com base em } 34 \text { itens em } \\
\text { escala Likert de pontos. }\end{array}$ & $\begin{array}{l}\text { O instrumento tem demonstrado } \\
\text { bons índices de validade } \\
\text { discriminante e concorrente e boa } \\
\text { confiabilidade teste-reteste, bem } \\
\text { como adequada consistência } \\
\text { interna. }\end{array}$ \\
\hline Escala de figuras de Stunkard & $\begin{array}{l}\text { A escala é composta de } \\
\text { nove figuras para cada } \\
\text { sexo que variam de } \\
\text { imagens de silhuetas muito } \\
\text { magras a muito gordas, } \\
\text { sendo usada para verificar } \\
\text { a insatisfação corporal. }\end{array}$ & $\begin{array}{c}\text { Esta medida de insatisfação atende } \\
\text { à concepção de que a insatisfação } \\
\text { corporal é dada pela distância entre } \\
\text { o corpo real e o corpo ideal. }\end{array}$ \\
\hline
\end{tabular}

Fonte: Adaptado de Guimarães et al. (2019)

Para Manochio et al. (2018) analisar e conhecer os indicadores de bom e mau prognóstico dos TA, acaba possibilitando a determinação com uma maior precisão a intensidade e o tipo de tratamento do paciente. Os transtornos alimentares podem ser tratados com fármacos 


\section{RECIMA21 - REVISTA CIENTÍFICA MULTIDISCIPLINAR ISSN 2675-6218}

REVISÃO DA LITERATURA SOBRE O TRATAMENTO FARMACOLÓGICO DOS TRANSTORNOS ALIMENTARES Jacqueline Gabriele Soares Ferreira, Chrislen Nascimento de Oliveira, Jhéssyca Glaycianne Souza das Chagas, Gleicy Kelly China Quemel, Natasha Costa da Rocha Galucio, Danilo Reymão Moreira

antidepressivos, agentes antiobesidade e anticonvulsivantes. No Gráfico 1 é possível visualizar o índice de prevalência dos distúrbios alimentares mais frequentemente avaliados nas literaturas utilizadas durante o estudo.

Gráfico 1: Distúrbios mais encontrados nos estudos selecionados

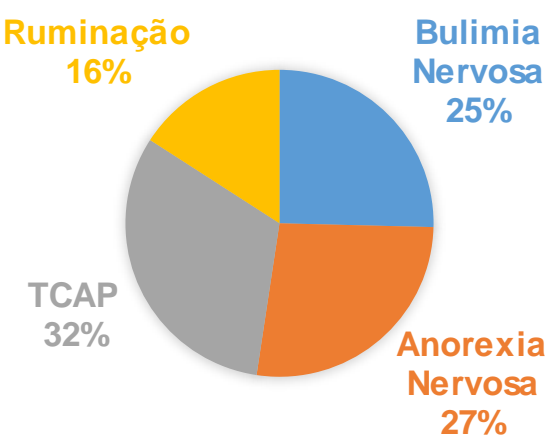

Fonte: Autores, 2021

O fármaco dimesilato de lisdexanfetamina já utilizado como tratamento farmacológico do transtorno de déficit de atenção e hiperatividade (TDAH) é uma nova opção de tratamento para pacientes com transtornos de Compulsão alimentar (BRASIL, 2021). Patrocínio et al. (2019) descreve que o mecanismo de ação acontece pelo bloqueio da receptação de neurotransmissores como a dopamina, que também aumenta a liberação de noradrenalina, fazendo com que o Sistema Nervoso Central seja estimulado e ocorra a diminuição da hiperatividade, regulando a vontade e o prazer em relação aos alimentos.

Cordas (2004) aborda o tratamento da compulsão alimentar, bulimia nervosa e Anorexia nervosa com o uso de fármacos e placebos, o estudo utilizou a Imipramina, uns antidepressivo tricíclicos que atua aumentando a quantidade de mediadores químicos como a noradrenalina e serotonina no cérebro, tem ação nos receptores alfa-adrenérgicos, histamínicos, colinérgicos e também bloqueia o sítio ativo de serotonina, contudo ela não apresentou mudanças no quadro clínico dos pacientes durante o estudo. A sibutramina um agente antiobesidade que atua como um inibidor seletivo de receptação de serotonina, noradrenalina e dopamina foi outro fármaco utilizado no estudo, mostrou-se eficaz para o tratamento da compulsão alimentar. Já a fluoxetina, é um antidepressivo responsável pela inibição seletiva da receptação de serotonina, esse neurotransmissor age sobre a regulação do humor, das emoções, do sono e do apetite. Mostrou efeito muito favorável na redução de episódios compulsivos e também na redução de peso.

O gráfico 2 , demostra o quantitativo do uso desses fármacos em relação à escolha da terapia mais adequada ao tratamento. 


\section{RECIMA21 - REVISTA CIENTÍFICA MULTIDISCIPLINAR ISSN 2675-6218}

REVISÃO DA LITERATURA SOBRE O TRATAMENTO FARMACOLÓGICO DOS TRANSTORNOS ALIMENTARES Jacqueline Gabriele Soares Ferreira, Chrislen Nascimento de Oliveira, Jhéssyca Glaycianne Souza das Chagas, Gleicy Kelly China Quemel, Natasha Costa da Rocha Galucio, Danilo Reymão Moreira

Gráfico 2 - Fármacos mais utilizados para o tratamento dos transtornos alimentares

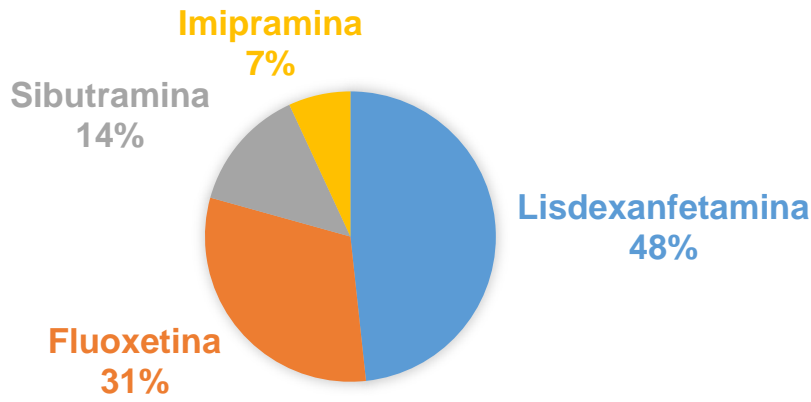

Fonte: Autoras,2021.

Outra possível terapia medicamentosa que pode ser utilizada no tratamento de TA está descrita por Sakae, Suka e Yanagisawa (2020), o polaprezinco, sendo um complexo de zinco-Lcarnosina disposto a reduzir significamente os episódios de compulsão alimentar. Sua ação farmacológica se dá a partir da modulação de neurotransmissores como o glutamato, já que o zinco se liga aos receptores de glutamato ( $\mathrm{N}$-metil-D-aspartato), servindo como agente modulador inibitório, já a L-carnosina tem propriedades anti-inflamatória, capaz de reduzir a excitotoxicidade da glicose, gerando uma regulação positiva nós transportadores de glutamato.

Com relação aos fatores de risco, para Fortes, Morgado e Pereira (2013) esses fatores estão ligados ao comportamento alimentar inadequado estão diretamente correlacionados a pressões externas, alguns comportamentos como, práticas de autoindução de regurgitação, restrições patológicas alimentares, uso de substâncias com caráter diurético e laxativos utilizados com um único propósito, obter a perda de peso.

Já Amoras et al. (2010) considera que essas práticas e comportamento são síndromes parciais, cogitando que estes sintomas são os principais fatores de risco para o desencadeamento de transtornos de comportamento alimentar (TCA), podendo acarretar uma série de sintomas e sequelas nos dentes e nos tecidos bucais, com erosões, hipersensibilidade, assim como o aumento de glândulas entre outras alterações patológicas, em casos mais severos pode comprometer seriamente a vida desde indivíduo.

Dentre tanto, Leal (2013) descreve que além dos sintomas clássicos de TA que são encontrados, outros comportamentos de risco considerados menos graves como algumas práticas de dietas ditas saudáveis visando garantir o controle sobre o peso corporal, o jejum ou a ingestão de uma quantidade mínima de alimento, pular refeições, usar suplementos como substitutos da alimentação ou o uso de medicações para emagrecer, podem ser tão nocivas à saúde quanto os sintomas clássicos dos transtornos alimentares, além das complicações já distas a cima, é possível encontrar outros tipos de alterações clínicas, como pode-se observar no Quadro 3. 


\section{RECIMA21 - REVISTA CIENTÍFICA MULTIDISCIPLINAR ISSN 2675-6218}

REVISÃO DA LITERATURA SOBRE O TRATAMENTO FARMACOLÓGICO DOS TRANSTORNOS ALIMENTARES Jacqueline Gabriele Soares Ferreira, Chrislen Nascimento de Oliveira, Jhéssyca Glaycianne Souza das Chagas, Gleicy Kelly China Quemel, Natasha Costa da Rocha Galucio, Danilo Reymão Moreira

Quadro 3 - Complicações clínicas que podem aparecer em pacientes com transtornos alimentares.

\begin{tabular}{|c|c|}
\hline Complicações Clínicas & Características Clínico-laboratoriais \\
\hline Metabólicas & $\begin{array}{l}\text { Hipocalemia } \\
\text { Hiponatremia } \\
\text { Hipernatremia } \\
\text { Hipomagnesia }\end{array}$ \\
\hline Hiperfosfatemia & $\begin{array}{l}\text { Hipoglicemia } \\
\text { Hipercolesterolemia } \\
\text { Alcalose metabólica } \\
\text { Acidose metabólica }\end{array}$ \\
\hline Neurológicas & $\begin{array}{l}\text { Alargamento dos sulcos cerebrais } \\
\text { Dilatação dos ventrículos } \\
\text { Atrofia cerebral (reversível) }\end{array}$ \\
\hline Oftalmológica & $\begin{array}{c}\text { Catarata } \\
\text { Atrofia do nervo óptico } \\
\text { Degeneração da retina }\end{array}$ \\
\hline Gastrointestinais & $\begin{array}{c}\text { Esofagite } \\
\text { Hematêmese } \\
\text { Retardo do esvaziamento gástrico } \\
\text { Redução da motilidade intestinal } \\
\text { Constipação } \\
\text { Prolapso retal } \\
\text { Dilatação gástrica } \\
\text { Alteração da função hepática } \\
\text { Hipertrofia das glândulas parótidas e submandibulares }\end{array}$ \\
\hline Renais & $\begin{array}{c}\text { Cálculos renais } \\
\text { Insuficiência renal }\end{array}$ \\
\hline Bucomaxilares e cutâneos & $\begin{array}{c}\text { Cáries dentárias } \\
\text { Ressecamento cutâneo, palidez } \\
\text { Sinal e Russel }\end{array}$ \\
\hline Pulmonares & $\begin{array}{c}\text { Taquicardia } \\
\text { Bradicardia } \\
\text { Edema pulmonar } \\
\text { Pneumomediastino }\end{array}$ \\
\hline Hematológica & $\begin{array}{l}\text { Anemia } \\
\text { Leucopenia } \\
\text { Trombocitopenia } \\
\text { Neutropenia }\end{array}$ \\
\hline
\end{tabular}

Fonte: Adaptado de Alckmin-Carvalho et al. (2013) e Cândido, Carmo, Pereira (2014)

Campos e Haack (2012) relataram que os transtornos alimentares são quadros psiquiátricos que afetam mais o sexo feminino, devido uma somatória de questões de cunho biológico, ligadas a personalidade e experiências individuais de cada paciente, gerando assim alterações emocionais e comportamentais, podendo evoluir a distúrbios menstruais, osteoporose e até mesmo arritmia cardíaca.

Já para Rodrigues et al. (2020) o transtorno alimentar é caracterizado por uma perturbação persistente na alimentação que resulta na absorção de alimentos inadequados, comprometendo assim a saúde dessas pessoas. O consumo excessivo de alimentos industrializados e de alto índice de gordura e açucares faz com que ocorram leves alterações nos micronutrientes gerando uma carência de ferro e uma possível anemia entre esses pacientes.

RECIMA21 - Ciências Exatas e da Terra, Sociais, da Saúde, Humanas e Engenharia/Tecnologia 


\section{RECIMA21 - REVISTA CIENTÍFICA MULTIDISCIPLINAR ISSN 2675-6218}

REVISÃO DA LITERATURA SOBRE O TRATAMENTO FARMACOLÓGICO DOS TRANSTORNOS ALIMENTARES Jacqueline Gabriele Soares Ferreira, Chrislen Nascimento de Oliveira, Jhéssyca Glaycianne Souza das Chagas, Gleicy Kelly China Quemel, Natasha Costa da Rocha Galucio, Danilo Reymão Moreira

Para Xião et al. (2019) a Covid-19 está associada à piora dos quadros de transtornos ligados a alimentação em pacientes com predisposições a está doença, o isolamento social proveniente da pandemia causada pelo Corona vírus ocasionou mudança na rotina do mundo todo, agravando distúrbios psicopatológicos, afetando a estabilidade emocional de muitos indivíduos.

\section{CONCLUSÃO}

A partir do levantamento dos estudos científicos observou-se que os transtornos de compulsão alimentar estão relacionados às comorbidades psicopatológicas; já os quadros depressivos e de ansiedade tem sido associado à Síndrome do Comer Muito, podendo ser influenciada pela provável concordância com a Perturbação de Ingestão Compulsiva.

Sendo assim, foi possível perceber que o atual mundo em que vivemos, impõe padrões estéticos que por fim acabam influenciando nos comportamentos psicológicos, promovendo um aumento nos quadros da depressão principalmente no caso dos indivíduos obesos, esta pressão imposta pela sociedade quando associada aos possíveis fatores genéticos e fisiológicos, aumentam os riscos de uma maior incidência de transtornos alimentares.

Dessa forma, faz-se necessário apontar a importância do papel do farmacêutico neste contexto, o profissional farmacêutico se encontra envolvido na fase do tratamento deste paciente, podendo orientar e acompanhar o mesmo em busca de uma estabilidade emocional, assim como de outros profissionais da saúde.

\section{REFERÊNCIAS}

ALCKMIN-CARVALHO, F. A. et al. Anorexia nervosa: diagnóstico, mudanças no perfil e tratamento. Pediatria Moderna, v. 49, n. 7, p. 296-299, 2013.

ALMEIDA, C. C.; ASSUMPÇÃO, A. A. A eficácia do mindful eating para transtornos alimentares e obesidade: revisão integrativa. Pretextos - Revista da Graduação em Psicologia da PUC Minas, v. 3, n. 6, p. 25-36, 2018.

AMORAS, D. R. et al. Caracterização dos transtornos alimentares e suas implicações na cavidade bucal. Rev. odontol., v. 39, 2010.

ARAÚJO, A. S. et al. Avaliação do consumo alimentar em pacientes com diagnóstico de depressão e/ou ansiedade. Revista Referencias em Saúde da Faculdade Estácio de Sá de Goiás-RRSFESGO., v. 03, n. 1, p. 18-26, 2020.

BARDIN, L. Análise de conteúdo. Traduzido por Luís Antero Reto, Augusto Pinheiro. São Paulo: Edições 70, 2011. (Tradução de: L'Analyse de Contenu).

BITTENCOURT, A. C. P.; FONTENELE, L. B. Uma análise psicanalítica da compulsão e da impulsão a partir da perspectiva do gozo e do ato. Cad. psicanal., v. 35, n. 28, p. 183-202, 2013.

BLOC, L. G. et al. Transtorno de compulsão alimentar: revisão sistemática da literatura. Rev.

Psicol. Saúde, Campo Grande, v. 11, n. 1, p. 3-17, 2019. 


\section{RECIMA21 - REVISTA CIENTÍFICA MULTIDISCIPLINAR ISSN 2675-6218}

REVISÃO DA LITERATURA SOBRE O TRATAMENTO FARMACOLÓGICO DOS TRANSTORNOS ALIMENTARES Jacqueline Gabriele Soares Ferreira, Chrislen Nascimento de Oliveira, Jhéssyca Glaycianne Souza das Chagas, Gleicy Kelly China Quemel, Natasha Costa da Rocha Galucio, Danilo Reymão Moreira

BRASIL. Relatório de recomendação. Dimesilato de lisdexanfetamina para indivíduos adultos com Transtorno do Déficit de Atenção com Hiperatividade. Brasília, DF: Ministério da Saúde, $2021 . \quad$ Disponível em: http://conitec.gov.br/images/Relatorios/2021/20210602 Relatorio 610 Lisdexanfetamina TDAH P 20.pdf. Acesso em: 06 nov. 2021.

CAMPOS, J. G. C.; HAACK, A. Anorexia e bulimia: aspectos clínicos e drogas habitualmente usadas no seu tratamento medicamentoso. Com. Ciências Saúde., v. 23, n. 3, p. 253-262, 2012.

CÂNDIDO, A. P. C.; CARMO, C. C.; PEREIRA, P. M. de L. Transtornos alimentares: uma revisão dos aspectos etiológicos e das principais complicações clínicas. HU Revista, v. 40, n. 3 e 4, 2015.

CAUDURO, G. N.; PACHECO, J. T. B.; PAZ, G. M. Avaliação e intervenção no transtorno da compulsão alimentar (tca): uma revisão sistemática. Psico, v. 49, n. 4, p. 384-394, 2018.

COMIN, F. S.; SANTOS, M. A. Psicoterapia como estratégia de tratamento dos transtornos alimentares: análise crítica do conhecimento produzido. Estudos de Psicologia, v. 29, Supl., p. 851s-863s, 2012.

CORDAS, T. A. Transtornos alimentares: classificação e diagnóstico. Revista de psiquiatria clínica, v. 31, n. 4, p. 154-157, 2004.

COSTA, M. B.; MELNIK, T. Efetividade de intervenções psicossociais em transtornos alimentares: um panorama das revisões sistemáticas Cochrane. Einstein, São Paulo, v. 14, n. 2, p. 235-77, 2016.

DE AZEVEDO, A. P.; DOS SANTOS, C. C.; DA FONSECA, D. C. Transtorno da compulsão alimentar periódica. Rev. Psiq. Clin., v. 31, n. 4, p. 170-172, 2004.

DE MELO, D. O.; DE CASTRO. A contribuição do farmacêutico para a promoção do acesso e uso racional de medicamentos essenciais no SUS. Ciência \& Saúde Coletiva., v. 22, n.1, p. 235-244, 2017.

DOS ANJOS, I. L. P. et al. Distúrbio alimentar, compulsivo e afetivo: uma revisão bibliográfica acerca da associação. Revista de Saúde, v. 11, n. 2, p. 60-64, 2020.

DUARTE, M. Q. et al. COVID-19 e os impactos na saúde mental: uma amostra do Rio Grande do Sul- Brasil. Ciência \& Saúde Coletiva, v. 25, n. 9, p. 3401-3411, 2020.

FORTES, L. S.; MORGADO, F. F. R.; FERREIRA, M. E. C. Fatores associados ao comportamento alimentar inadequado em adolescentes escolares. Rev Psiq Clín., v. 40, n. 2, p. 59-64, 2013.

GUIMARÃES, I. S. et. al. Transtornos Alimnetares. Boletim Sociedade Brasileira de Neuropsicologia (SBNp), São Paulo, SP, v. 2, n. 10, p. 1-30, out. 2019. Disponível em: https://sbnpbrasil.com.br/wp-content/uploads/2019/11/24-Boletim Out-2019.pdf. Acesso em: 06 nov. 2011.

LEAL, G. V. S. et al. O que é comportamento de risco para transtornos alimentares em adolescentes? J Bras Psiquiatr, v. 62, n. 1, p. 62-75, 2013.

LEAL, G. V. S. Fatores associados ao comportamento de risco para transtornos alimentares em adolescentes na cidade de São Paulo. 2013. Tese (Doutorado em Nutrição em Saúde Pública) - Faculdade de Saúde Pública, Universidade de São Paulo, São Paulo, 2013.

MANOCHIO, M. G. et al. Tratamento dos transtornos alimentares: perfil dos pacientes e desfecho do seguimento. Rev. Interdisciplin. Promoç. Saúde, v. 1, n. 1, p. 32-40, 2018. 


\section{RECIMA21 - REVISTA CIENTÍFICA MULTIDISCIPLINAR ISSN 2675-6218}

REVISÃO DA LITERATURA SOBRE O TRATAMENTO FARMACOLÓGICO DOS TRANSTORNOS ALIMENTARES Jacqueline Gabriele Soares Ferreira, Chrislen Nascimento de Oliveira, Jhéssyca Glaycianne Souza das Chagas, Gleicy Kelly China Quemel, Natasha Costa da Rocha Galucio, Danilo Reymão Moreira
Gata

MENDES, K. D. S.; SILVEIRA, R. C. C. P.; GALVÃO, C. M. Revisão integrativa: método de pesquisa para a incorporação de evidências na saúde e na enfermagem. Texto Contexto Enferm, v. 17, n. 4, p. 758-64, 2008.

PALAVRAS, M. A. et al. Uma revisão dos estudos latino-americanos sobre o transtorno da compulsão alimentar periódica. Revista Brasileira de Psiquiatria, v. 33, Supl. I, 2011.

PATROCÍNIO, M. C. A. et al. Psicofarmacologia e psiquiatria geral: para graduandos e generalistas [recurso eletrônico]. Fortaleza: EdUnichristus, 2019. 33 p.

PINHEIRO, N. P. Classificação e Diagnóstico de Transtornos Alimentares na Infância: Nem DSM, nem CID-10. Psicol. pesq., v. 5, n. 1, p. 61-67, 2011.

RAMOS, T. M. B.; PEDRÃO, J. L. Acolhimento e Vínculo em um Serviço de Assistência a Portadores de Transtornos Alimentares. Paidéia., v. 23, n. 54, p. 113-120, 2013.

RODRIGUES, B. B. et al. Aprendendo com o imprevisível: saúde mental dos universitários e educação médica na pandemia de covid-19. Rev Bras Educ Med., v. 44, n. 1, p. 1-5, 2020.

RODRIGUES, G. F. P. Atenção farmacêutica no tratamento de transtornos alimentares. Revista Acadêmica Conecta (FASF)., v. 3, n. 1, 2018.

SAKAE, K.; SUKA, M.; YANAGISAWA, A. H. Polaprezinc (Zinc-L-Carnosine Complex) as an Add-on Therapy for Binge Eating Disorder and Bulimia Nervosa, and the Possible Involvement of Zinc Deficiency in These Conditions. Journal of Clinical Psychopharmacology., v. 40, n. 6, 2020.

SILVA, A. M. B. et al. Jovens Insatisfeitos com a Imagem Corporal: Estresse, Autoestima e Problemas Alimentares. Psico - USF, v. 23, n. 3, p. 483-495, 2018.

STATNICK, M. A.; et al. A Novel Nociceptin Receptor Antagonist LY2940094 Inhibits Excessive Feeding Behavior in Rodents: A Possible Mechanism for the Treatment of Binge Eating Disorder. J Pharmacol Exp Ther., v. 356, n. 2, p. 493-502, 2016.

VIANNA, M. V. Compulsão Alimentar \& Cirurgia Bariátrica: aspectos da fome que o bisturi não alcança. 2018. 186f. Tese (doutorado) - Pontifícia Universidade Católica do Rio de Janeiro, Departamento de Psicologia, 2018.

WERNECK, A. O. et al. Lifestyle behaviors changes during the COVID-19 pandemic quarantine among 6,881 Brazilian adults with depression and 35,143 without depression. Ciência \& Saúde Coletiva, v. 25, Supl. 2, p. 4151-4156, 2020.

XIAO, H. et al. The Effects of Social Support on Sleep Quality of Medical Staff Treating Patients with Coronavirus Disease 2019 (COVID-19) in January and February 2020 in China. Medical science monitor: international medical journal of experimental and clinical research, v. 26, p. e923549, 2020. 